\title{
Efeito da abundância dos adultos sobre as ninfas de Gerromorpha (Heteroptera) em riachos de Cerrado no estado de Goiás
}

\author{
Kevin Cardoso ${ }^{1}$ \\ Kesley Gadelha ${ }^{1}$ \\ Gustavo Ferreira ${ }^{1}$ \\ Kenned Silva ${ }^{1}$ \\ Paulo Augusto Oliveira ${ }^{1}$ \\ Karen Moy ${ }^{2}$ \\ Thiago B. Vieira ${ }^{2}$ \\ Karina Dias-Silva ${ }^{2 *}$ \\ Universidade Federal do Pará \\ ${ }^{1}$ Graduação em Licenciatura em Ciências Biológicas, Faculdade de Ciências Biológicas \\ ${ }^{2}$ Programa de Pós-Graduação em Biodiversidade e Conservação \\ Rua Cel. José Porfírio, Esplanada do Xingu, CEP 68.372-040, Altamira - PA, Brasi \\ * Autor para correspondência \\ diassilvakarina@gmail.com
}

Submetido em 20/09/2018

Aceito para publicação em 09/04/2019

\section{Resumo}

Entender como as espécies interagem entre si e como o ambiente afeta as espécies é um dos objetivos que os ecólogos ainda buscam responder. Diante disso os nossos objetivos foram avaliar o efeito (i) da abundância de adultos de Gerromorpha, (ii) da integridade de habitat e (iii) das condições morfológicas (largura, profundidade e declividade) do riacho sobre a abundância de ninfas de Heteroptera semiaquáticos (Gerromorpha). As coletas foram realizadas em 21 córregos na região de Barro Alto e Niquelândia, no estado de Goiás. A abundância de adultos de Gerromorpha exerce efeito positivo sobre a abundância de ninfas. A integridade de habitat, as variáveis morfológicas do canal (largura, profundidade e a declividade) não exerceram efeito sobre a abundância de ninfas e de adultos de Gerromorpha. Em ambientes tropicais, adultos e ninfas de Gerromorpha coexistem sem prejuízos, e as variáveis ambientais não exercem efeito sobre a abundância de ninfas de Gerromorpha. A proteção das ninfas é importante do ponto de vista populacional, pois garante a manutenção e o crescimento das populações presentes na comunidade e, consequentemente, evita extinções locais de espécies.

Palavras-chave: Canibalismo; Coexistência; Insetos aquáticos; Integridade de habitat; Predação 


\section{Abstract}

Effect of adult abundance on nymphs of Gerromorpha (Heteroptera) in Cerrado streams in the state of Goiás. How species interact with each other and how the environment affects species are subjects that ecologists still need to better understand. Our objectives were to evaluate the effect of (i) the abundance of Gerromorpha adults, (ii) habitat integrity, and (iii) morphological conditions (width, depth and slope) of streams on the abundance of nymphs of semiaquatic Heteroptera (Gerromorpha). Sampling was carried in 21 streams in the region of Barro Alto and Niquelândia, in the state of Goiás. The abundance of Gerromorpha adults has a positive effect on the abundance of nymphs. The morphological conditions (width, depth and slope) and habitat integrity had no effect on the abundance of Gerromorpha nymphs and adults. In tropical environments, adults and nymphs of Gerromorpha coexist without harming each other and environmental variables have no effect on the abundance of the nymphs. The protection of nymphs is important from a population point of view, since it ensures the maintenance and growth of populations in a community and, consequently, prevents local extinctions of species.

Key words: Aquatic insects; Cannibalism; Coexistence; Habitat integrity; Predation

\section{Introdução}

Os Heteroptera aquáticos compreendem três infraordens (SCHUH; SLATER, 1995; POLHEMUS; POLHEMUS, 2008): os Nepomorpha, os Leptopodomorpha e os Gerromorpha. Os Nepomorpha compreendem os percevejos aquáticos e vivem geralmente associados aos substratos bentônicos e à coluna d'água. Os Leptopodomorpha e os Gerromorpha compreendem os percevejos habitantes da superfície de corpos d'água, ambientes ripícolas e até áreas intertidais, sendo assim denominados "semiaquáticos" (SPENCE; ANDERSEN, 1994; SCHUH; SLATER, 1995; NIESER; MELO, 1997). Os representantes da infraordem Gerromorpha são insetos neustônicos, visto que geralmente ficam sobre a superfície da água em ambientes continentais lênticos e lóticos (SOUZA et al., 2006).

Possuem desenvolvimento hemimetábolo, passando pelas fases imatura, ninfa e adulta $(\mathrm{SCHUH}$; SLATER, 1995). A vantagem adaptativa desse tipo de desenvolvimento é que muitas ninfas possuem hábitos alimentares similares aos dos adultos e vivem em ambientes bastante diferentes dos adultos evitando assim a competição intraespecífica (HUTCHINSON, 1959). Essa subordem é composta principalmente por insetos predadores que se alimentam de pequenos insetos, podendo as espécies maiores se alimentar de pequenos peixes com exceção de algumas famílias, por exemplo Corixidae, que se alimenta de algas e detritos (NIESER;
MELO, 1997; POLHEMUS; POLHEMUS, 2008) sendo que os adultos e ninfas compartilham os mesmos hábitos alimentares e comportamentais (NUMMELIN et al., 1984), sendo comum observarmos o canibalismo. Uma estratégia comportamental, adotada pelas ninfas para diminuir e evitar o canibalismo, é evitar locais onde os adultos se encontram em grande abundância (NUMMELIN et al., 1984; SPENCE; CÁRCAMO, 1991).

As condições morfológicas e de integridade do ambiente afetam as comunidades aquáticas, uma vez que influenciam no sucesso da reprodução e na obtenção de alimento (COSSINS; CRAWFORD, 2005). Mudanças nessas variáveis ambientais afetam negativamente algumas espécies mais sensíveis e acabam propiciando um aumento de abundância das espécies mais tolerantes (CUNHA et al., 2015; CUNHA; JUEN, 2017; GIEHL et al., 2018). Essas alterações diminuem a qualidade do habitat tanto na questão física (substrato e sombreamento) quanto na questão das variáveis físico-químicas, tais como $\mathrm{pH}$, oxigênio dissolvido e temperatura da água (ALLAN; CASTILLO, 2007).

A integridade física é um importante componente dos ecossistemas aquáticos e quando essa integridade é alterada pode comprometer todas as comunidades aquáticas. A retirada da vegetação ciliar, assim como a modificação nos riachos, exerce efeito negativo sobre os Gerromorpha (DIAS-SILVA et al., 2010; VIEIRA et al., 2015). Assim, trabalhos que visem avaliar o efeito da 
integridade sobre as ninfas de Gerromorpha contribuirão para o conhecimento de como as alterações ambientais podem afetar a persistências dessas comunidades nos riachos que são dependentes da vegetação ripária.

Desse modo, visto que os Gerromorpha podem ser afetados pelas condições locais do habitat e que as ninfas e os adultos são predadores e compartilham os mesmos recursos, o objetivo do trabalho foi avaliar: (i) o efeito da abundância de adultos, (ii) o efeito da integridade de habitat e (iii) variáveis de morfologia do canal (largura, profundidade e declividade) do riacho sobre a abundância de ninfas de Gerromorpha.

\section{Material e Métodos}

\section{Área de estudo}

As coletas foram realizadas em 21 córregos na região de Barro Alto e Niquelândia no ano de 2011 no estado de Goiás (Figura 1; Tabela 1). A região apresenta dois períodos bem distintos em relação à pluviosidade. A classificação de Köppen Aw (Cwa) (PEEL, et al., 2007) descreve um clima com temperaturas elevadas, chuvas no verão (outubro-março) e seca no inverno (abril-setembro) (NIMER, 1989).

FIGURA 1: Localização espacial dos córregos amostrados no município de Barro Alto e Niquelândia - GO, em 2011.

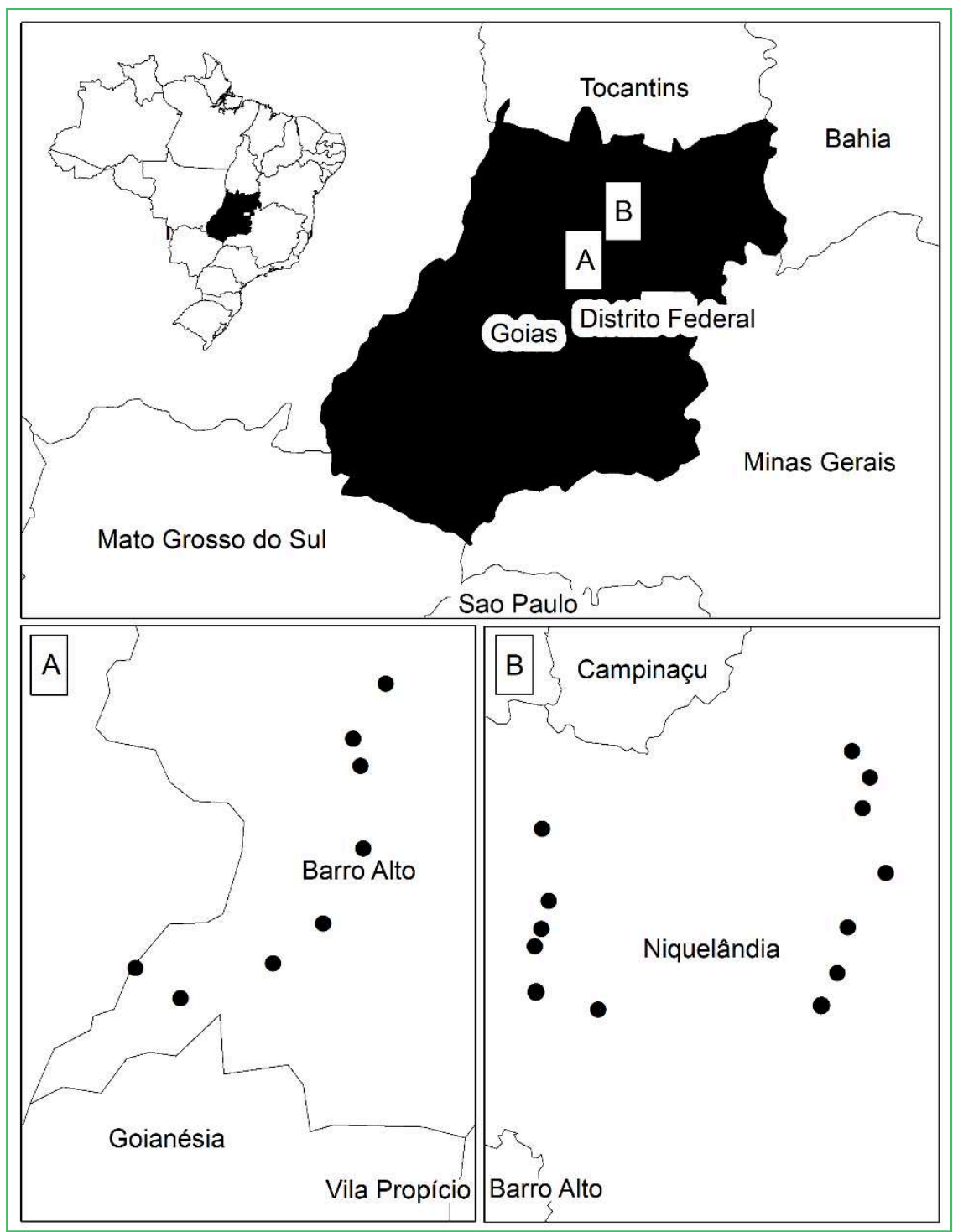


TABELA 1: Pontos amostrados, coordenadas geográficas, municípios, Índice de Integridade Física do Habitat - IIH, largura e profundidade (Prof.) dos córregos amostrados em Niquelândia e Barro Alto, GO, Brasil, em 2011.

\begin{tabular}{|c|c|c|c|c|c|c|c|}
\hline \multirow{2}{*}{ Riachos } & \multirow{2}{*}{ Município } & \multirow{2}{*}{ Data } & \multicolumn{2}{|c|}{ Coordenadas } & \multirow{2}{*}{ IIH } & \multirow{2}{*}{$\begin{array}{l}\text { Largura } \\
\text { (m) }\end{array}$} & \multirow{2}{*}{$\begin{array}{l}\text { Prof. } \\
(\mathrm{cm})\end{array}$} \\
\hline & & & Latitude (S) & Longitude (W) & & & \\
\hline $\mathrm{P} 01$ & Niquelândia & $6 / 14 / 2011$ & -14.183 & -48.350 & 0,53 & 4,08 & 23,40 \\
\hline P02 & Niquelândia & $6 / 14 / 2011$ & -14.215 & -48.328 & 0,43 & 1,94 & 15,53 \\
\hline $\mathrm{P} 03$ & Niquelândia & $6 / 15 / 2011$ & -14.252 & -48.337 & 0,39 & 4,02 & 26,74 \\
\hline P04 & Niquelândia & $6 / 15 / 2011$ & -14.331 & -48.309 & 0,45 & 2,44 & 19,70 \\
\hline P05 & Niquelândia & $6 / 16 / 2011$ & -14.277 & -48.726 & 0,34 & 3,20 & 18,33 \\
\hline P06 & Niquelândia & $6 / 16 / 2011$ & -14.277 & -48.726 & 0,42 & 4,22 & 27,77 \\
\hline P07 & Niquelândia & $6 / 17 / 2011$ & -14.365 & -48.718 & 0,37 & 3,92 & 19,30 \\
\hline P08 & Niquelândia & $6 / 17 / 2011$ & -14.399 & -48.727 & 0,39 & 2,48 & 10,13 \\
\hline P09 & Niquelândia & 6/17/2011 & -14.420 & -48.735 & 0,34 & 1,70 & 22,70 \\
\hline P10 & Niquelândia & $6 / 18 / 2011$ & -14.397 & -48.355 & 0,31 & 2,56 & 26,40 \\
\hline P11 & Niquelândia & $6 / 18 / 2011$ & -14.453 & -48.368 & 0,30 & 2,32 & 14,83 \\
\hline P12 & Niquelândia & 6/19/2011 & -14.497 & -48.658 & 0,31 & 1,72 & 13,47 \\
\hline P13 & Niquelândia & $7 / 19 / 2011$ & -15.078 & -48.965 & 0,58 & 5,26 & 23,00 \\
\hline P14 & Barro Alto & $7 / 20 / 2011$ & -15.094 & -48.985 & 0,59 & 3,04 & 18,00 \\
\hline P15 & Barro Alto & $7 / 20 / 2011$ & -15.048 & -48.949 & 0,44 & 1,86 & 5,40 \\
\hline P16 & Barro Alto & $7 / 21 / 2011$ & -15.015 & -48.950 & 0,39 & 4,09 & 13,20 \\
\hline P17 & Barro Alto & $7 / 21 / 2011$ & -15.004 & -48.953 & 0,35 & 2,70 & 18,33 \\
\hline P18 & Barro Alto & $7 / 21 / 2011$ & -14.982 & -48.940 & 0,28 & 1,47 & 15,27 \\
\hline P19 & Barro Alto & $7 / 22 / 2011$ & -15.132 & -47.042 & 0,46 & 3,46 & 15,07 \\
\hline P20 & Barro Alto & $7 / 22 / 2011$ & -15.096 & -49.040 & 0,48 & 1,70 & 16,53 \\
\hline $\mathrm{P} 21$ & Barro Alto & $7 / 23 / 2011$ & -15.108 & -49.022 & 0,53 & 2,45 & 32,45 \\
\hline
\end{tabular}

\section{Coleta de dados em campo}

As coletas dos Gerromorpha seguiram a metodologia proposta por Cabette et al. (2010), sendo que em cada córrego foi demarcado um transecto de 100 $\mathrm{m}$ lineares, com amostragem a cada $5 \mathrm{~m}$, totalizando 20 amostras por córrego. Cada amostra foi composta por três subamostras, uma em cada margem e uma no centro do segmento, de $5 \mathrm{~m}$. O material coletado foi triado e conservado em álcool a 85\%, depositado no Laboratório de Ecologia Teórica e Síntese-LETS, da Universidade Federal de Goiás, e identificado com auxílio de chaves taxonômicas.

Em cada corpo d'água foram realizadas medidas de largura, profundidade e declividade. A largura média do canal $(\mathrm{m})$ foi calculada a partir de cinco medidas equidistantes $(20 \mathrm{~m})$ ao longo do transecto de $100 \mathrm{~m}$.
A profundidade média do canal (m) foi calculada, nos mesmos locais onde a largura foi aferida, sendo feitas três sondagens em cada uma das cinco medidas, uma no centro e uma em cada margem do canal. Adeclividade foi medida com um auxílio de uma mangueira transparente de $10 \mathrm{~m}$ contendo água em seu interior e duas réguas. Com as extremidades da mangueira posicionada acima da lâmina d'água foi medida a altura em que a bolha de ar ficava da lâmina d'água e posteriormente a menor altura foi subtraída da maior altura, gerando assim os valores de declividade. Esse procedimento foi repetido dez vezes em cada riacho e quando não foi possível fazer a medida a cada $10 \mathrm{~m}$, devido a irregularidades no canal (presença de rochas e troncos), realizamos pelo menos três medidas no riacho; posteriormente foi calculada a declividade média. 
A integridade física do ambiente foi avaliada com o Índice de Integridade de Habitat (IIH) proposto por Nessimian et al. (2008). O IIH é composto por doze itens que buscam descrever as condições ambientais do riacho e do seu entorno, tais como padrão de uso da terra adjacente à vegetação ribeirinha; largura da mata ciliar e estado de preservação; avaliação da mata ciliar dentro de uma faixa de $10 \mathrm{~m}$. Além disso, avalia as condições do canal quanto ao tipo de sedimento e à presença de dispositivos de retenção (tais como bancos de areia, rochas e madeiras); estrutura e desgaste dos barrancos marginais do rio; caracterização do leito do rio quanto ao substrato, vegetação aquática, detritos e disposição das áreas de corredeiras, poções e meandros. Cada item (questão) é composto de quatro ou seis alternativas ordenadas e resultam em um único valor que varia de 0 a 1 , em que valores próximos a 0 representam riachos degradados e valores próximos a 1 riachos conservados.

\section{Análises estatísticas}

Para avaliar o efeito da abundância de adultos e do IIH sobre a abundância de ninfas, realizamos regressões lineares simples (ZAR, 2010) entre a abundância de ninfas e de adultos e entre a abundância de ninfas e o IIH. Para avaliar o efeito da morfologia do canal (largura, profundidade e declividade) sobre a abundância das ninfas e de adultos, utilizamos uma regressão linear múltipla (ZAR, 2010).

\section{Resultados}

Foram coletados 4982 indivíduos de Gerromorpha, em que 2.109 eram ninfas e 2.873 adultos. Observamos que apenas a abundância de adultos de Gerromorpha exerce efeito sobre a abundância de ninfas $\left(\mathrm{F}_{(1,18)=} 7,209\right.$; $\mathrm{p}=0,015)$. A abundância de adultos explica $33,4 \%$ da variação observada nas ninfas e o seu efeito é positivo (Figura 2), aproximadamente uma ninfa é adicionada ao sistema para cada dois adultos observados. As variáveis de morfologia do canal (largura, profundidade e declividade) $\left.\left(\mathrm{F}_{3,14}\right)=1,268 ; \mathrm{R}^{2}=0,045 ; \mathrm{p}=0,323\right)$ e o IIH $\left.\left(\mathrm{F}_{1,18}\right)=0,679 ; \mathrm{p}=0,420\right)$ não exerceram efeito sobre a abundância de ninfas nem sobre os adultos de
Gerromorpha $\left.\left(\mathrm{F}_{3,14}\right)=0,260 ; \mathrm{R}^{2}=0,046 ; \mathrm{p}=0,8526\right) \mathrm{e}$ $\left(\mathrm{F}\left({ }_{1,18}\right)=0,172 ; \mathrm{p}=0,682\right)$.

FIGURA 2: Efeito da abundância de adultos sobre a abundância de ninfas de Gerromorpha no estado de Goiás.

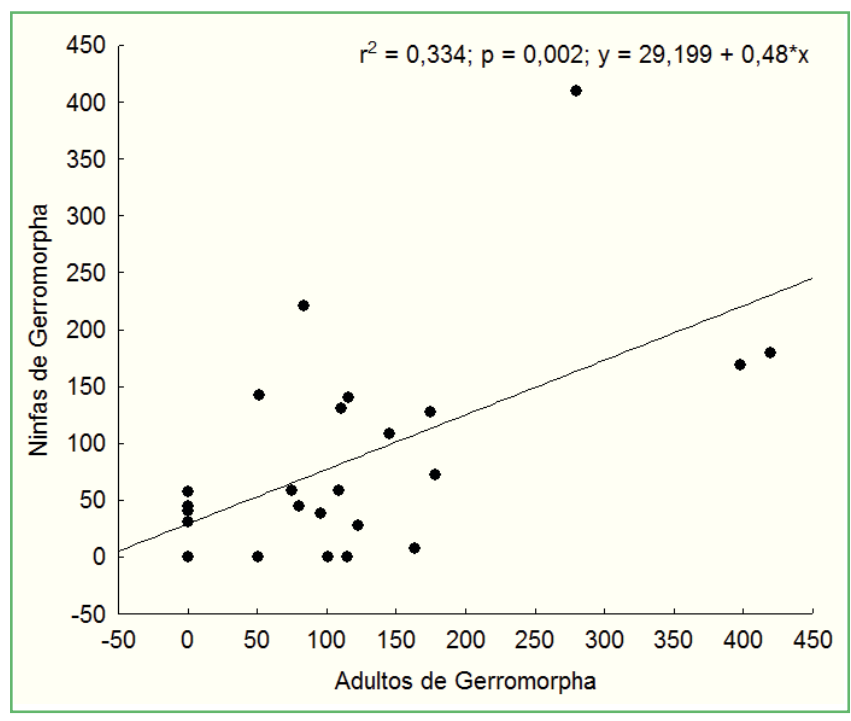

\section{Discussão}

Em nosso estudo, ao contrário do esperado pela literatura, a abundância de ninfas é diretamente proporcional à abundância dos adultos. Sabemos que a disponibilidade de recursos é um fator que regula o canibalismo em Heteroptera (SPENCE; CÁRCAMO, 1991). Assim, em ambientes onde a oferta de alimento não é escassa, espécies que partilham os mesmos recursos podem coexistir. Deste modo, uma explicação para essa relação positiva é a oferta constante de alimento nos ambientes tropicais. A predição de que ninfas evitam locais com altas densidade de adultos é derivada de observações em ambientes temperados (SCHUH; SLATER, 1995), locais que apresentam períodos de restrição alimentar devido aos períodos de baixas temperaturas, uma vez que o canibalismo nesse grupo é comum na escassez de alimento e dependente da disponibilidade de habitat (NUMMELIN et al., 1984; NUMMELIN, 1989; SPENCE; CÁRCAMO, 1991; CÁRCAMO; SPENCE, 1994).

Em ambientes tropicais, adultos e ninfas de Gerromorpha coexistem sem prejuízos, sendo que a morfologia do canal e o IIH não exercem efeito sobre a abundância de Gerromorpha, isso podendo estar 
relacionado com o hábito de vida e o hábito alimentar do grupo. Os integrantes do grupo são indivíduos neustônicos, ou seja, vivem sobre a superfície da água e são predadores, não selecionando a espécie das suas presas. Deste modo, as condições ambientais, sejam elas físicas, químicas ou físico-químicas, acabam por não influenciar diretamente esses indivíduos (DIASSILVA et al., 2010; VIEIRA et al., 2015; GIEHL et al., 2018). Pode ser que a composição das espécies de presa mude e, no entanto, a oferta de alimento continue a existir. $\mathrm{O}$ mesmo argumento pode ser utilizado para o IIH, pois o índice é composto por questões que descrevem as condições físicas internas do riacho, dos barrancos e de vegetação ciliar. Apesar de o IIH ter variado $(0,30-0,59)$, os riachos apresentavam vegetação ciliar e barrancos consideravelmente estáveis, duas características importantes para os Gerromorpha, pois essas variáveis propiciam a presença de raízes, substratos que proporcionam habitat para muitas espécies (DIASSILVA et al., 2013). A presença de vegetação ciliar e consequentemente de presas é um fator que influencia nas taxas de canibalismo, sendo que a qualidade de presa pode aumentar ou diminuir o canibalismo em Heteroptera aquáticos (PANIZZI; PARRA, 2012). Além disso, o grupo apresenta plasticidade a pequenas alterações ambientais (DIAS-SILVA et al., 2010).

Desse modo, a relação direta entre ninfas e adultos encontrada no estudo pode ter se dado devido à forma de amostragem, que é passiva, assim, temos mais ninfas em um determinado lugar devido ao local ter maior número de indivíduos adultos de Gerromorpha. Apesar da não relação entre morfologia do canal e IIH com a abundância de ninfas, estudos posteriores que investiguem os fatores que estruturam a comunidade (presença/ausência e abundância das espécies) são necessários. Entender como as comunidades se estruturam no tempo e no espaço e como os fatores ambientais determinam essa estrutura é um fator chave na restauração das comunidades degradadas. Além disso, estudos que visem identificar quais os fatores estruturam a comunidade adulta ainda são necessários, uma vez que não sabemos se a mesma resposta passiva seria observada em adultos. Outra pergunta a ser respondida diz respeito às respostas de cada espécie presente na comunidade, uma vez que espécies de diferentes tamanhos tendem a preferir presas diferentes, recurso que pode ser variável em função das condições ambientais existentes.

Assim, nosso estudo mostra que adultos e ninfas coexistem em riachos, indicando assim que, ao se preservar os adultos de Gerromorpha, acabamos protegendo as ninfas e consequentemente os serviços providos por esses predadores. A proteção das ninfas é importante do ponto do vista populacional, pois garante a manutenção e o crescimento das populações presentes na comunidade e consequentemente evitam extinções locais de espécies.

\section{Agradecimentos}

Os autores agradecem a empresa de mineração Anglo American, pelo apoio logístico e pelo financiamento. À Fundação de Amparo a Pesquisa, FUNAPE-UFG, pelo gerenciamento do projeto. KCRL e GFS agradecem a Universidade Federal do Pará (UFPA) pela bolsa PIBIC. KGF agradece a UFPA pela bolsa PIBEX. TBV agradece a bolsa de doutorado CAPES (001).

\section{Referências}

ALLAN, J. D.; CASTILLO, M. M. Stream Ecology: structure and function of running waters. 2. ed. New York: Chapman and Hall. 2007. 444 p.

CABETTE, H. S. R.; GIEHL, N. F. S.; DIAS-SILVA, K.; JUEN, L.; BATISTA, J. B. Distribuição de Nepomorpha e Gerromorpha (Insecta: Heteroptera) da Bacia Hidrográfica do Rio Suiá-Miçu, MT: riqueza relacionada à qualidade da água e do hábitat. In: SANTOS, J. E.; GALBIATI, C.; MOSCHINI, L. E. (Org.). Gestão e educação ambiental: água, biodiversidade e cultura. São Carlos: RiMa, 2010. p.113-137.

CÁRCAMO, H.; SPENCE, J. Kin Discrimination and Cannibalism in Water Striders (Heteroptera: Gerridae): Another Look. Oikos, Copenhagen, v. 70, n. 3, p. 412-416, 1994.

COSSINS, A. R.; CRAWFORD, D. L. Fish as models for environmental genomics. Nature, London, v. 6, p. 324-331, 2005.

CUNHA, E. J.; MONTAG, L. F. A.; JUEN, L. (2015). Oil palm crops effects on environmental integrity of Amazonian streams and Heteropteran (Hemiptera) species diversity. Ecological Indicators, v. 52, p. 422-429.

CUNHA, E. J.; JUEN, L. 2017. Impacts of oil palm plantations on changes in environmental heterogeneity and Heteroptera (Gerromorpha and Nepomorpha) diversity. Journal Insect and Conservation, v. 21, p. 111-119.

CUNHA, E. J.; MONTAG, L. F. A.; JUEN, L. (2015). Oil palm crops effects on environmental integrity of Amazonian streams and 
Heteropteran (Hemiptera) species diversity. Ecological Indicators, v. 52, p. 422-429.

CUNHA, E. J.; JUEN, L. (2017). Impacts of oil palm plantations on changes in environmental heterogeneity and Heteroptera (Gerromorpha and Nepomorpha) diversity. Journal Insect and Conservation, v. 21, p. 111-119.

CUNHA, E. J.; MONTAG, L. F. A.; JUEN L. (2015). Oil palm crops effects on environmental integrity of Amazonian streams and Heteropteran (Hemiptera) species diversity. Ecological Indicators, v. 52 , p. $422-429$

CUNHA, E. J.; JUEN, L. (2017). Impacts of oil palm plantations on changes in environmental heterogeneity and Heteroptera (Gerromorpha and Nepomorpha) diversity. Journal Insect and Conservation, v. 21, p. 111-119.

CUNHA, E. J.; MONTAG, L. F. A.; JUEN L. (2015). Oil palm crops effects on environmental integrity of Amazonian streams and Heteropteran (Hemiptera) species diversity. Ecological Indicators, v. 52p. 422-429.

CUNHA, E. J.; JUEN, L. Impacts of oil palm plantations on changes in environmental heterogeneity and Heteroptera (Gerromorpha and Nepomorpha) diversity. Journal Insect and Conservation, London, v. 21, p. 111-119, 2017.

CUNHA, E. J.; MONTAG, L. F. A.; JUEN, L. Oil palm crops effects on environmental integrity of Amazonian streams and Heteropteran (Hemiptera) species diversity. Ecological Indicators, Amsterdam, v. 52, p. 422-429, 2015.

DIAS-SILVA, K.; CABETTE, H. S. R.; GIEHL, N. F. S.; JUEN, L. Distribuição de Heteroptera aquáticos (Insecta) em diferentes tipos de substratos de córregos do Cerrado Matogrossense. EntomoBrasilis, Vassouras, v. 6, n. 2, p. 132-140, 2013.

DIAS-SILVA, K.; CABETTE, H. R. S.; JUEN, L.; DE MARCO JR., $\mathrm{P}$. The influence of habitat integrity and physical-chemical water variables on the structure of aquatic and semi-aquatic Heteroptera. Zoologia, Curitiba, v. 27, p. 918-930, 2010.

GIEHL, N. F. S.; BRASIL, L. S.; DIAS-SILVA, K.; NOGUEIRA, D. S.; CABETTE, H. S. R. Environmental Thresholds of Nepomorpha in Cerrado Streams, Brazilian Savannah. Neotropical Entomology, Londrina, v. 48, n. 2, p. 186-196, 2018.

HUTCHINSON, G. E. Homage to Santa Rosalia or why are there so many kinds of animals? The American Naturalist, Chicago, v. 93, n. 870, p. 145-159, 1959.

NESSIMIAM, J. L.; VENTICINQUE, E.; ZUANON, J.; DE MARCO JR., P.; GORDO, M.; FIDELIS, L.; BATISTA, J. D.; JUEN, L. Land use, habitat integrity, and aquatic insect assemblages in Central Amazonian streams. Hydrobiologia, Brussels, v. 614, p. $117-131,2008$.
NIESER, N.; MELO, A. L. Os heterópteros aquáticos de Minas Gerais. Belo Horizonte: Editora UFMG, 1997. 177 p.

NIMER, E. Climatologia do Brasil. Rio de Janeiro: IBGE, 1989. $422 \mathrm{p}$.

NUMMELIN, M. Cannibalism in waterstriders (Heteroptera: Gerridae): is there kin recognition? Oikos, Copenhagen, v. 56, p. 87-90, 1989 .

NUMMELIN, M.; VEPSÄLÄINEN, K.; SPENCE, J. R. Habitat partitioning among developmental stages of waterstriders (Heteroptera: Gerridae). Oikos, Copenhagen, v. 42, p. 267-275, 1984.

PANIZZI, A. R.; PARRA, J. R. P. Insect bioecology and nutrition for integrated pest management. New York: CRC Press, 2012. $750 \mathrm{p}$.

PEEL, M. C.; FINLAYSON, B. L.; MCMAHON, T. A. Updated world map of the Köppen-Geiger climate classification. Hydrology and Earth System Sciences, Göttingen, v. 11, n. 5, p. 1633-1644, 2007.

POLHEMUS, J. T.; POLHEMUS, D. A. Global diversity of true bugs (Heteroptera: Insecta) in freshwater. Hydrobiologia, Brussels, v. 595, p. 379-391, 2008.

SCHUH, R. T.; SLATER, J. A. True bugs of the World (Hemiptera: Heteroptera): classification and natural history. Ithaca and London: Cornell University Press, 1995. 336 p.

SOUZA, M. A. A. de; MELO, A. L. de; VIANNA, G. J. C. Heterópteros aquáticos oriundos do município de Mariana, MG. Neotropical Entomology, Londrina, v. 35, n. 6, p. 803-810, 2006.

SPENCE, J. R.; ANDERSEN, N. M. Biology of waterstriders: interactions between systematics and ecology. Annual Review of Entomology, Palo Alto, v. 39, p. 97-124, 1994.

SPENCE, R. S.; CÁRCAMO, H. A. Effects of cannibalism and intraguild predation on pondskaters (Gerridae). Oikos, Copenhagen, v. 62, p. 333-341, 1991.

VIEIRA, T. B.; DIAS-SILVA, K.; PACÍFICO E. S. Effects of riparian vegetation integrity on fish and heteroptera communities. Applied Ecology and Environmental Research, Budapest, v. 13, n. 1, p. 53-65, 2015.

ZAR, J. H. Biostatistical analysis. New Jersey: Prentice Hall, 2010. 944 p. 